\title{
Manifestation of Central Diabetes Insipidus in a Patient with Thyroid Storm
}

\author{
Akiko Nakamichi, Kazuki Ocho, Kosuke Oka, Miho Yasuda, Kou Hasegawa, \\ Masaya Iwamuro, Mikako Obika, Kammei Rai and Fumio Otsuka
}

\begin{abstract}
:
We herein report a case of central diabetes insipidus complicated with thyroid storm. A middle-aged woman who was receiving treatment for Graves' disease suddenly complained of polydipsia, polyuria and general fatigue. Laboratory tests showed hyperthyroidism, hypernatremia, hypoosmolar urine and a decreased plasma vasopressin level. The occurrence of central diabetes insipidus with hyperthyroidism was revealed on the basis of pituitary magnetic resonance imaging, a water deprivation test and a desmopressin test. The clinical co-existence of diabetes insipidus and hyperthyroidism is very rare; however, the complication should be considered when hypernatremia and/or dehydration progress in patients with Graves's disease as a common autoimmune-related etiology.
\end{abstract}

Key words: central diabetes insipidus, Graves' disease, hyperthyroidism, lymphocytic infundibuloneurohypophysitis and thyroid crisis

(Intern Med 57: 1939-1942, 2018)

(DOI: 10.2169/internalmedicine.0063-17)

\section{Introduction}

Thyroid crisis, also called thyroid storm, and central diabetes insipidus (DI) are both rare endocrine disorders (1). It has been reported that the incidence of thyroid storm in hospitalized patients was approximately 0.20 per 100,000 per year and that thyroid storm can be induced by stressful events such as surgery, trauma, infection and parturition (2). In contrast, the most common causes of central DI are idiopathic, primary or secondary tumors and infiltrative disease. Lymphocytic infundibulo-neurohypophysitis (LINH), which induces inflammatory reactions in the infundibulum and neurohypophysis, has been recognized as the main etiology of central DI (3). Although these two conditions are clinically distinct, autoimmune mechanisms are commonly involved in these diseases.

We herein report a rare complication of central DI in a patient with Graves' disease who simultaneously developed thyroid crisis.

\section{Case Report}

A 51-year-old woman with hyperthyroidism had been treated with oral thiamazole for 5 years. She had no remarkable medical history except for autoimmune thyroiditis. Her family and social histories were not remarkable. On her regular checkup at 2 months before admission, her thyroid hormone levels were found to be moderately high (free thyroxin: FT4, $3.38 \mathrm{ng} / \mathrm{dL}$; and free triiodothyronine: FT3, 9.74 $\mathrm{pg} / \mathrm{mL}$ ). However, after self-discontinuance of taking thiamazole for a month for an unmentioned reason, she suddenly felt thirsty and developed tachycardia and general fatigue with mental restlessness. She was therefore admitted for control of overt hyperthyroidism. Her height was 161 $\mathrm{cm}$, and her weight was $40.5 \mathrm{~kg}$, with a body mass index (BMI) of 15.6. Her body temperature was high $\left(38.3^{\circ} \mathrm{C}\right)$ with an increased pulse rate of $148 \mathrm{bpm}$. Her blood pressure was $121 / 83 \mathrm{mmHg}$, and her urinary volume was increased to $>3$ L/day. On the day of admission, she fell into delirium and severe emotional disturbance. Laboratory data showed elevation of the serum sodium levels $(149-160 \mathrm{mmol} / \mathrm{L})$.

Department of General Medicine, Okayama University Graduate School of Medicine, Dentistry, and Pharmaceutical Sciences, Japan Received: August 11, 2017; Accepted: December 10, 2017; Advance Publication by J-STAGE: February 28, 2018 Correspondence to Dr. Fumio Otsuka, fumiotsu@md.okayama-u.ac.jp 

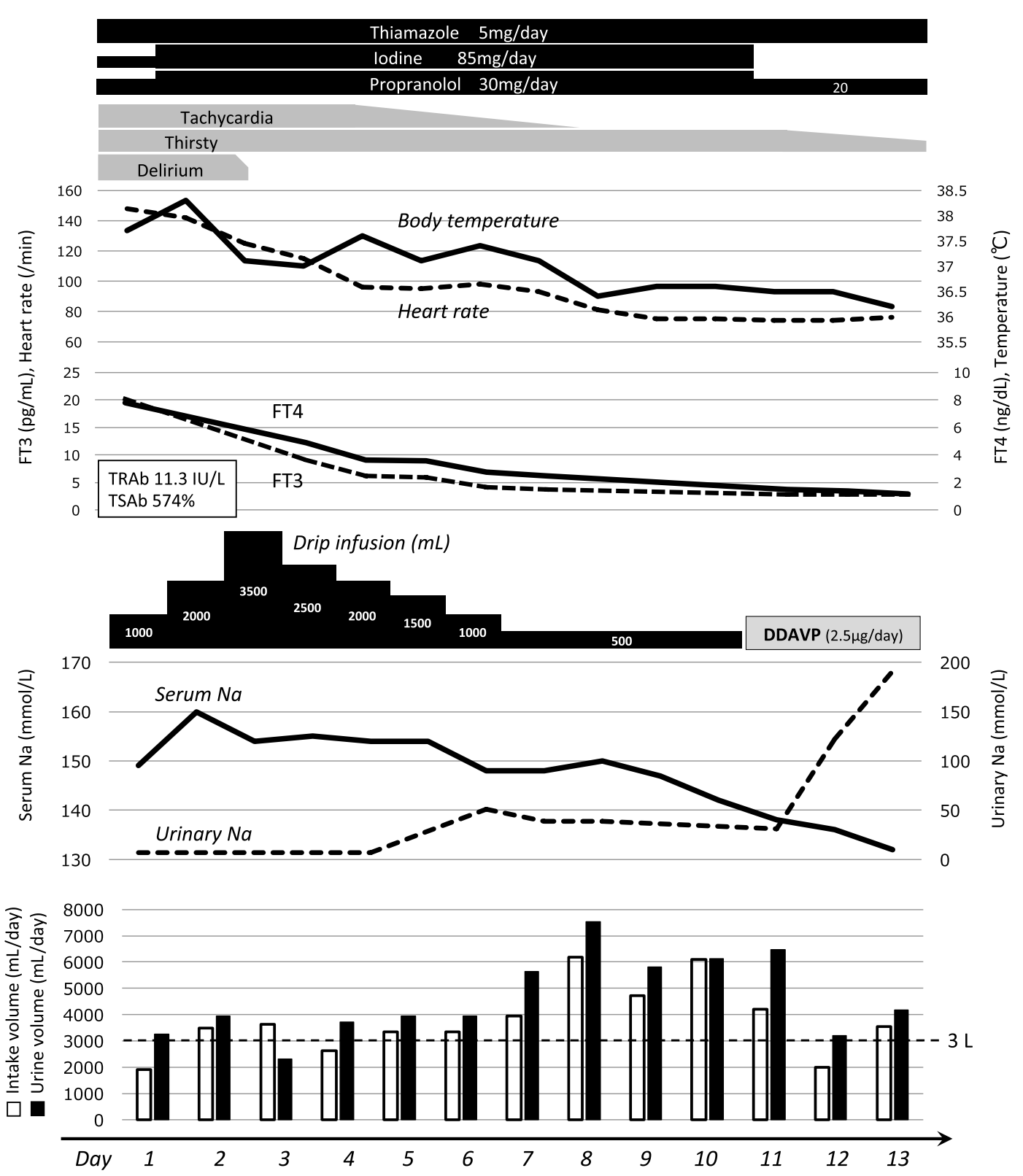

Figure 1. The clinical course. After the diagnosis of thyroid storm, propranolol and iodine were administered in addition to thiamazole for treatment. The delirium, fever and tachycardia improved at an early stage, and the levels of thyroid hormones (FT4 and FT3) gradually normalized. After starting treatment with desmopressin acetate (DDAVP) based on the diagnosis of central DI, the serum sodium (Na) levels normalized, and the DI symptoms, including polydipsia and polyuria, disappeared without drip infusion.

The serum osmolarity was high $(315 \mathrm{mOsm} / \mathrm{kg})$, while the urine osmolarity was low (90 mOsm/ $\mathrm{kg}$ ). Endocrine workup uncovered her severe hyperthyroidism as follows: FT4, > $7.77 \mathrm{ng} / \mathrm{dL}$; FT3, $20.13 \mathrm{pg} / \mathrm{mL}$; and thyroxin, $<0.01 \mu \mathrm{U} / \mathrm{mL}$. Thyroxin receptor antibodies were also highly positive (TRAb, 11.3 IU/L; TSAb, 574\%), and ultrasound showed a diffusely enlarged thyroid gland with an increased blood flow.

Upon the diagnosis of thyroid crisis, iodione and propranolol were administered in addition to thiamazole (Fig. 1, upper panel). Her delirium, fever and tachycardia gradually improved, and thyroid hormones were also normalized in nine days. However, regardless of the continuation of drip infusion, she still felt persistently thirsty and had polydipsia and polyuria. The serum sodium level (approximately 150 $\mathrm{mmol} / \mathrm{L}$ ) and osmolarity were still high, and the urine osmolarity had lowered, but the plasma arginine vasopressin (AVP) level had decreased (1.1 pg/mL) (Fig. 1, lower panel). A water deprivation test failed to increase the urinary osmolarity $(<100 \mathrm{mOsm} / \mathrm{kg})$, but the administration of desmopressin acetate (DDAVP) readily increased the urine osmolarity (82 to $307 \mathrm{mOsm} / \mathrm{kg}$ ) with a decrease in the urine volume (300 to $40 \mathrm{~mL} / \mathrm{h}$ ) (Fig. 1, lower panel). Her polydipsia, polyuria and psychiatric symptoms gradually improved after DDAVP treatment for two weeks. On serial magnetic resonance imaging (MRI), a highly intensified posterior sig- 


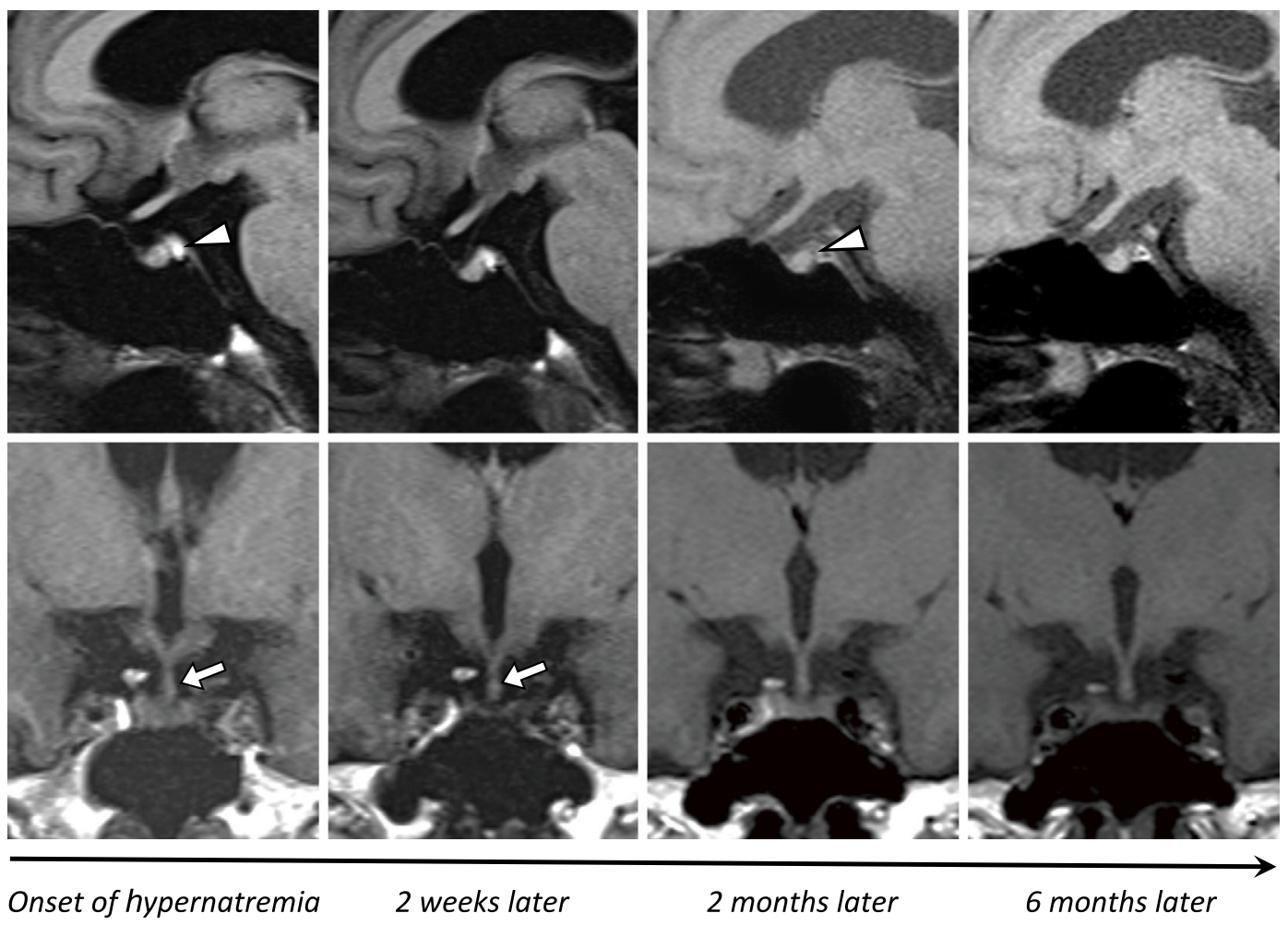

Figure 2. Pituitary MRI findings. Serial MRI showed a normal-shaped pituitary with gradual depletion of the high-intensity signal of the posterior lobe by T1-weighted MRI. The T1-weighted highintensity signal (arrowheads) was detected at the onset of polyuria, and the pituitary stalk was slightly thickened (arrows). At two months after the onset of DI, follow-up MRI showed a deficit in the T1weighted high-intensity signal in the posterior lobe (arrowheads), and the thickness of the pituitary stalk spontaneously diminished over two weeks (arrows).

nal on a T1-weighted image was detected at the onset of polyuria, and the size and shape of the pituitary gland were normal, but the pituitary stalk was slightly thickened (Fig. 2). At two months after the onset of clinical DI, follow-up MRI showed decreased T1 high-intensity signals in the posterior lobe, suggesting the depletion of AVP granules, and the thickness of the pituitary stalk spontaneously normalized after two weeks (Fig. 2). She continued to receive small doses of thiamazole and DDAVP, and her thyroid function and urine volume have been stably normalized.

\section{Discussion}

The present case showed the co-existence of central DI and hyperthyroidism. With thiamazole treatment for Graves' disease, she had sudden complaints of polydipsia, polyuria and general fatigue, and thyroid storm developed. The Burch and Wartofsky's score of thyrotoxicosis was 60 (>45), suggesting the occurrence of thyroid storm (4). The coexistence of central DI and hyperthyroidism is very rare, but this complication makes the control of both diseases difficult. The possibility of accompanying DI was not considered in the initial diagnosis of the patient's psychiatric changes. After admission, despite the continuation of drip infusion, the levels of serum sodium and osmolarity were not normalized, and the urine output exceeded 3 L/day. The patho- physiology of the central DI in the present case is unclear, since a biopsy was not performed, anti-pituitary antibody was negative, and the levels of immunoglobulin G4 (IgG4), angiotensin-converting enzyme (ACE) and lysozyme were normal.

Regarding the pathophysiology of complications of central DI and Graves' disease, Pivonello et al. suggested the significance of pre-existing autoimmunity in patients with idiopathic central DI (5). In their study, autoimmunity was associated with approximately one-third of patients with idiopathic central DI, in which the clinical features of autoimmune-related central DI are seen in younger patients with a clinical history of autoimmune diseases, such as autoimmune thyroiditis and type-I diabetes, and with radiological evidence of pituitary stalk thickening (5).

The pathogenesis of LINH, which causes central DI, has yet to be clarified. The histological features are polyclonal inflammatory cell infiltration with a $\mathrm{T}$ and $\mathrm{B}$ cell mixture, mature lymphocytes and some plasma cells (6). These features are likely to be seen in the posterior pituitary and pituitary stalk accompanying fibrotic or atrophic changes. Some cases of central DI caused by LINH may become self-limiting in terms of radiological and/or endocrinological changes $(6,7)$. Serial MRI in the present case also showed a spontaneously diminished thickening of the pituitary stalk despite a persistent lack of T1 high intensity of the posterior 
lobe. Recent research has also provided evidence that rabphilin-3A is a major autoantigen for LINH and thus might be useful as a biomarker for the diagnosis of central DI (8). Clinical relationships have been detected between LINH and other diseases, including autoimmune thyroiditis (9), systemic lupus erythematosus (10) and Behçet's disease (11) as well as a drug-induced case (12). However, the complication of DI secondary to thyroid storm has not been well documented.

A similar complicated case, but with a transient course of central DI, was reported by Yamazaki et al. (9) showed interesting findings that may prompt reconsideration of our case. A middle-aged man diagnosed with Graves' disease and central DI had received treatment with thiamazole. Of interest, DDAVP administration was able to be discontinued due to improvement of his Graves' disease. Yamazaki et al. reported that the recovery of the renal response to AVP and the immune-modulative effects of thiamazole, which inhibit prostaglandin-E2 resulting in sustaining the AVP action (9), might have contributed to the improvement of polyuria (9). In contrast, our case showed a distinct course demonstrating severe symptoms due to thyroid crisis and persistent central DI that required continuous DDAVP administration, even after the recovery from thyroid crisis. Considering the findings from the present and previous cases, we should therefore be alert for the possibility of spontaneous recovery from endogenous AVP depletion in cases like our own, although continuous DDAVP treatment has been required for longer than a year after the manifestation of central DI in some cases.

In conclusion, the present case shows that onset of central DI can be related to thyroid storm and that thyrotoxicosis may induce and/or exacerbate co-existing DI. Attention should be paid to this complication when hypernatremia and/or dehydration progress in patients with hyperthyroidism. The accumulation of similar cases is necessary to clarify the pathophysiology underlying the co-existence of these endocrine diseases.

The authors state that they have no Conflict of Interest (COI).

\section{References}

1. Sarlis NJ, Gourgiotis L. Thyroid emergencies. Rev Endocr Metab Disord 4: 129-136, 2003.

2. Akamizu T, Satoh T, Isozaki O, et al. Japan Thyroid Association. Diagnostic criteria, clinical features, and incidence of thyroid storm based on nationwide surveys. Thyroid 22: 661-679, 2012.

3. Imura H, Nakao K, Shimatsu A, et al. Lymphocytic infundibuloneurohypophysitis as a cause of central diabetes insipidus. $\mathrm{N}$ Engl J Med 329: 683-689, 1993.

4. Burch HB, Wartofsky L; Life-threatening thyrotoxicosis. Thyroid storm. Endocrinol Metab Clin North Am 22: 263-277, 1993.

5. Pivonello R, De Bellis A, Faggiano A, et al. Central diabetes insipidus and autoimmunity: relationship between the occurrence of antibodies to arginine vasopressin-secreting cells and clinical, immunological, and radiological features in a large cohort of patients with central diabetes insipidus of known and unknown etiology. $\mathrm{J}$ Clin Endocrinol Metab 88: 1629-1636, 2003.

6. Johnston PC, Chew LS, Hamrahian AH, Kennedy L. Lymphocytic infundibulo-neurohypophysitis: a clinical overview. Endocrine 50: 531-536, 2015.

7. Takahashi M, Otsuka F, Miyoshi T, Ogura T, Makino H. An elderly patient with transient diabetes insipidus associated with lymphocytic infundibulo-neurohypophysitis. Endocr J 46: 741-746, 1999.

8. Iwama S, Sugimura Y, Kiyota A, et al. Rabphilin-3A as a targeted autoantigen in lymphocytic infundibulo-neurohypophysitis. J Clin Endocrinol Metab 100: E946-E954, 2015.

9. Yamazaki M, Sato A, Nishio S, Uehara T, Komatsu M. Transient polyuria related to central diabetes insipidus caused by lymphocytic infundibulo-neurohypophysitis in a patient treated for Graves' disease. Intern Med 49: 1885-1890, 2010.

10. Hashimoto K, Asaba K, Tamura K, Takao T, Nakamura T. A case of lymphocytic infundibuloneurohypophysitis associated with systemic lupus erythematosus. Endocr J 49: 605-610, 2002.

11. Otsuka F, Amano T, Ogura T, Ota Z. Diabetes insipidus with Behçet's disease. Lancet 346: 1494-1495, 1995.

12. Otsuka F, Mizobuchi S, Morita K, et al. Postanesthetic polyuria attributable to central diabetes insipidus. Anesth Analg 85: 940-943, 1997.

The Internal Medicine is an Open Access article distributed under the Creative Commons Attribution-NonCommercial-NoDerivatives 4.0 International License. To view the details of this license, please visit (https://creativecommons.org/licenses/ by-nc-nd/4.0/).

(C) 2018 The Japanese Society of Internal Medicine Intern Med 57: 1939-1942, 2018 